wrapping the photographic plate. Later on they obtained a line of type AgKa - NiK abs. (Prof. M. N. Saha, in a private communication, has written to us that Messrs. Bhargava and Mukherjee have also found a line of the type AgKa-CuK $K_{\text {sbs. }}$ )

Cork (l.c.) reports a line $\mathrm{CuK} \alpha-\mathrm{Be} K a$ by passing copper Ka-radiation through Lindeman glass, which contains beryllium ( 0.9 per cent) among other constituents. As we have found in our experiments in carbon screen, Cork also finds that the intensity of this line does not increase by placing a beryllium screen in the path of the incident radiation. Further, non-appearance of any line modified by boron (which is another constituent of the Lindeman glass) might be due to the higher percentage of boron in the glass.

Lindsay (l.c.) has suggested that sometimes false bands appear on the photographic plate, due to the imperfection of the crystals. In our experiments the modified lines were obtained with both fixed and rotating erystals on a large number of plates. Generally, the crystal was rotated by hand about $2^{\circ}$ on each side of the expected line. The rate of rotation of the crystal was $0 \cdot 1^{\circ}$ in every five minutes. In some plates this rate was varied. The modified line appeared as usual in every case, and thus the question of rotation of the plate does not affect our results so far as the modified lines are concerned.

The intensity estimate ${ }^{7}$ is wrong, owing to an unfortunate mistake in comparing the blackness with a wrong plate. We have re-estimated the intensity of the modified lines and it appears to be of the order of 1 in 1000 (or even less) in comparison with the parent line. Agfa-Röntgen film is found to be more suitable for this type of investigation than ordinary plates.

B. B. RAY.

University College of Science,

92 Upper Circular Road, Calcutta, May 1.

1 Natore, May 17, June 7, Sept. 13, 1930.

Comptes rendus, Jan. 1931.

NATURE, Feb. 28, 1931

- Naturwissen., April 3, 1931

- Nature, Feb. 21, Feb. 28, 193

- NATuRE, June 7. 1930 ; Zeit.f. Phys., Bd. 66.

Zeit.f. Phys., Bd. 66 .

\section{Fine Structure of Raman Lines in Liquids.}

I HAVE investigated the Raman spectra of several liquids with a spectrograph of high dispersion specially built for this purpose. The spectrograph was placed in a thermostat in order to allow very long exposures without losing anything in definition, owing to variation in the refractive index of the glass prisms.

With this apparatus I have observed, for the first time, so far as I know, the isotope effect in Raman spectra. I have succeeded in measuring the isotope effect in carbon tetrachloride and in stannic chloride, among other substances. The observation confirms the analysis of the infra-red absorption spectra of this substance proposed by $\mathrm{Cl}$. Schaefer. ${ }^{1}$ The work will appear in detail in the Zeitschrift für Physik.

I have also obtained beautiful Raman spectra of a number of different liquids. The plates show that the Raman lines have a characteristic structure, resembling unresolved vibration-rotation bands. This confirms the suggestion recently proposed by A. Kastler ${ }^{2}$ that the rotations of the molecules in liquids are quantised.

Concentrated ammonia liquor gives a strong Raman band which is due to the $\mathrm{NH}_{3}$-molecule. This band shows a distinct rotation structure. It is composed of a strong, unresolved $Q$-branch, surrounded on both sides by a succession of lines, of which the strongest have a spacing corresponding to the formula

$$
\nu=3317 \cdot 4 \pm 19.87 \times J .
$$

Further, there is a number of weaker lines with an average spacing of $9 \cdot 7 \mathrm{~cm} .^{-1}$. The rotation structure of the band consists obviously of a strong $Q$-branch and two positive branches, a strong $R$-and a weaker $S$-branch. Of the negative branches, the $P$-branch is rather strong, while the $O$-branch seems to be very weak. The intensity of the lines has a maximum at $J=4-5$, as in the infra-red absorption spectrum.

The Raman band of water has a very complicated structure which, on addition of an electrolyte, becomes more distinct. In collaboration with Mr. Hugo Fredholm, I am extending systematic work in this direction.

I might finally mention a result which implies the possibility of a partial rotation of a molecule, as suggested by Bonino and Cella ${ }^{3}$ in the case of pinene. Toluene, $\mathrm{C}_{6} \mathrm{H}_{5} . \mathrm{CH}_{3}$, gives a number of Raman lines of which two, commonly aseribed to the vibration $\mathrm{C}-\mathrm{H}$, show rotation structure. The Raman band $\nu_{0}=3056.3 \mathrm{~cm}^{-1}$, has the simplest structure. It is composed of a strong, unresolved $Q$-branch, a strong negative and a weaker positive branch, corresponding to the formula

$$
\nu=3056 \cdot 3 \pm 7 \cdot 0 \times J .
$$

If these branches are interpreted as $P$ - and $R$ branches, the spacing leads to a value for the moment of inertia, $I=7.9 \times 10^{-40} \mathrm{gm}$. cm. ${ }^{2}$, which is in excellent agreement with the value to be expected for a rotating methyl group. Further details will be given elsewhere.

Chemical Laboratory of the University.

$$
\text { Copenhagen, Juity } 14 .
$$

A. Langseth.

1 Zeit. f. Phys., 60, 586; 1930.

Compt. rend., 192, $1032 ; 1931$

S NATORE, 12B, $915 ; 1930$

\section{Occurrence of Lithothamnion in the South Indian} Gretaceous.

WhILE examining some micro-sections of rocks from the Trichinopoly Cretaceous (South India), I have recognised the presence of Lithothamnion in several of the sections. This form has not been

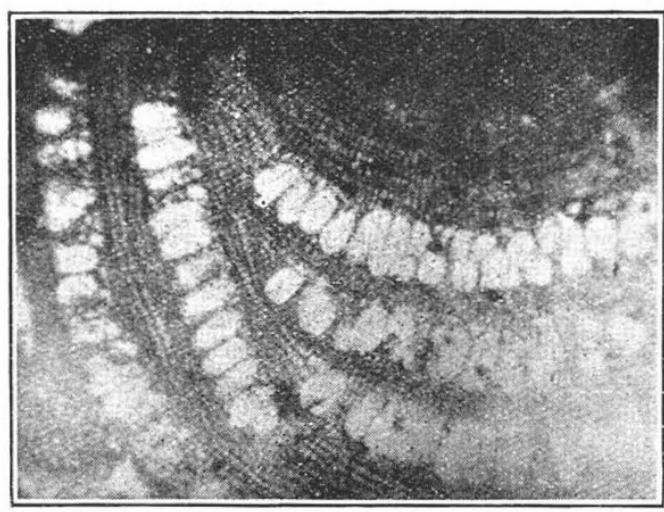

FIG. 1.-Archæolithothamnion sp ? $\times$ abont 25

reported before from this area; and except for a passing reference by Hayden ${ }^{1}$ to the occurrence of Lithothamnion in certain Cretaceous (?) beds of the Kampa system in Tibet, $I$ believe the present find is the first of its kind to be reported from the Cretaceous of any part of India.

In view of the great interest attached to this

No. 3223, Vor. 128] 